\title{
A Quantitative Study of the Expression of Unselected Lactose and Proline Markers in Escherichia coli K-12 Recombinants and in the Parent Strains
}

\author{
By S. BAUMBERG* \\ Physical Chemistry Laboratory, Oxford University
}

(Received 12 October 1965)

\begin{abstract}
SUMMARY
The standard methods of scoring for unselected markers the recombinant clones arising from crosses between various substrains of Escherichia coli k-12 do not easily allow recognition of types intermediate between or slightly deviating from the parental phenotypes. The theories of Hinshelwood and his school suggest the general possibility of a continuous series of such intermediates, which have in fact been reported among the progeny of certain drug-resistant $\times$ drug-sensitive crosses. To examine whether similar types result with other kinds of unselected marker, crosses were performed in which the parents differed in their ability to utilize lactose (lac character) and/or to grow in the absence of proline (pro character), as well as in other characteristics utilizable as selected markers. Growth of recombinant and parental isolates on minimal media containing lactose as carbon source, or lacking proline, was then characterized quantitatively. No recombinants with intermediate pro character were found, and only one with intermediate lac character. However, in some cases the recombinants differed in lac or pro character from either parent in other ways. One donor strain, proline-requiring $\left(\mathrm{pro}^{-}\right)$and unable to utilize lactose $\left(\mathrm{lac}^{-}\right)$, yielded $\mathrm{pro}^{-}$and $\mathrm{lac}^{-}$recombinants whose eventual growth in prolineless and lactose media respectively was greatly retarded by comparison with the donor parent. This donor strain was also crossed with a $\mathrm{pro}^{+} \mathrm{lac}^{+}$ recipient which had been 'trained' to grow in minimal lactose medium with optimal growth rate. In lactose media, $\mathrm{lac}^{+}$recombinants all grew at the optimal rate, while $l a c^{-}$recombinants showed growth still retarded as compared to the lac $^{-}$parent, but considerably more rapid than similar recombinants from the cross involving an untrained $l a c^{-}$recipient.
\end{abstract}

\section{INTRODUCTION}

The recombinant clones arising in bacterial crosses are usually scored for unselected markers by streaking a suspension of the recombinant, freed from parental contamination, on media so constituted as to permit differences in various characteristics between the parent strains to show as visible differences in growth. For instance, one may recognize in this way differences in ability to grow in the absence of particular growth factors or in the presence of antibacterial agents, or in ability to ferment particular carbon sources as reflected in the colour of streaks forming on indicator media (J. Lederberg, 1947). However, with these qualitative methods,

\footnotetext{
* Present address: Institute of Microbiology, Rutgers, The State University, New Brunswick, New Jersey, U.S.A.
} 
there may be a tendency to classify recombinants as either ' + ' or ' - ' for every character, possible types intermediate between or deviating only slightly from the parental being inserted into one or the other category.

In the case of parent strains one of which has acquired the ability to grow at high concentrations of an antibiotic, resistance to which is acquired by a 'multi-step' process (Demerec, 1948), quantitative methods have been developed to characterize the level of resistance shown by recombinants (Cavalli \& Maccacaro, 1950, 1952; Bartlett \& Hinshelwood, 1959). In these cases it has been shown that the recombinants present a complete spectrum of degrees of resistance from that of the sensitive to that of the resistant parent. With most other types of unselected marker, however, such quantitative methods have not been used, probably in view of the assumption that, where the difference between parent strains can be attributed to differing activities of a single enzyme, these different activities reflect distinct configurations of the genetic material, either of which may appear in recombinants, but between which no continuous series of intermediates can be formed.

The views of Dean \& Hinshelwood $(1964 a, b)$, who suppose that variations in phenotype may reflect changes in the values of self-perpetuating reaction cycles in the bacterial cell, suggest an alternative approach to bacterial heredity. These values would be capable of continuous variation; hence one might expect that, on introduction of material from one strain into those of another, as occurs in conjugation, the recombinants (by whatever process they may originate) might show continuous variation in behaviour.

This report records the results of some experiments in which the characters of parents and recombinants were estimated quantitatively in terms of characteristics of growth. Two characters were used: ability to utilize lactose as carbon source and ability to grow in the absence of proline. The above comments on the possibility of overlooking intermediate types apply to selected as well as to unselected markers, and hence the growth characteristics of recombinant colonies on the selecting media were carefully examined. A check was also made of possible growth on the selecting medium of either of the parent strains alone (involving reversion to the wild type of a character used for counter-selection, or mutation to streptomycin resistance).

\section{Organisms.}

\section{METHODS}

The strains used are listed in Table 1 . Strains $\mathrm{Hfr}$ H 59, $\mathrm{Hfr}$ R 1, 75 and Y-10 were obtained from Dr W. Hayes and his colleagues of the M.R.C. Microbial Genetics Research Unit, London, to whom we express our thanks.

Media.

Nutrient media. Nutrient broth and agar were prepared from the 'Oxoid No. 2' and 'Oxoid' dried products respectively.

Minimal media. The 'salts' solution contained, in 1 l. of water: $\mathrm{Na}_{2} \mathrm{HPO}_{4} \cdot \mathbf{1 2 H}_{2} \mathrm{O}$, 10 g.; $\mathrm{KH}_{2} \mathrm{PO}_{4}, 1 \cdot 85$ g.; $\left(\mathrm{NH}_{4}\right)_{2} \mathrm{SO}_{4}, 1 \cdot 56$ g.; $\mathrm{MgSO}_{4} .7 \mathrm{H}_{2} \mathrm{O}, 62 \cdot 5 \mathrm{mg}$.; $\mathrm{FeSO}_{4} .7 \mathrm{H}_{2} \mathrm{O}$, $0.315 \mathrm{mg}$.

Fully supplemented minimal ('FSM') 'limiting-glucose' media, for routine 
subculturing, contained $15 \mathrm{ml}$. salts solution, $2 \mathrm{ml}$. glucose solution (5 g./l.), then $2 \mathrm{ml}$. threonine or proline solution (1.35 g./l.), $1 \mathrm{ml}$. leucine or methionine solution $(\mathbf{1} \cdot 35 \mathrm{~g} . / \mathrm{l})$, and $0.2 \mathrm{ml}$. thiamine hydrochloride solution $(450 \mathrm{mg} . / \mathrm{l}$.), as necessary. Media for testing growth characteristics contained $15 \mathrm{ml}$. salts solution, $5 \mathrm{ml}$. glucose solution ( $5 \mathrm{~g}$. $/ \mathrm{l}$.) or lactose (10 g./l.), then $1 \mathrm{ml}$. threonine or proline solution (9 g./l.), $1 \mathrm{ml}$. leucine or methionine solution, and $0.2 \mathrm{ml}$. thiamine hydrochloride solution (strengths as before), as necessary. L-amino acids were used throughout. 'These media are 'complete' (see below) for all growth factors. Streptomycin sulphate, the product of Glaxo Labs. Ltd., was added to a final concentration of $200 \mathrm{units} / \mathrm{ml}$.

Solid minimal media were made up by solidifying the above media with $1.25 \%$ Davis 'Standard Agar'.

\title{
Table 1. Characteristics of Escherichia coli strains used
}

Strain
designation
$\mathbf{5 9}$
$\mathbf{R} 1$
$\mathbf{7 5}$
$\mathbf{7 5 1}$
$\mathbf{Y}-10$
$\mathbf{8 0 R}$

Mating type
Hfr Hayes
Hfr Reeves 1
F- $^{-}$
F $^{+}$
F $^{-}$
F- $^{-}$

\author{
Genotype \\ Thi- lac- $\left(z_{1}^{-}\right) S^{8}$ \\ Prototroph $\mathrm{lac}^{+} \mathrm{S}^{\mathrm{s}}$ \\ Met $^{-}$pro $^{-}$lac $^{-}\left(y^{-}\right) S^{r}$ \\ As 75 \\ Thr- leu- thi- lac $+S^{s}$ \\ As Y-10, but $S^{r}$
}

Abbreviations used. Mating types: Hfr, high-frequency donor; $\mathbf{F}^{+}$, low-frequency donor; $\mathbf{F}^{-}$, recipient. Markers : $\mathrm{Thi}^{-}, \mathrm{met}^{-}, \mathrm{pro}^{-}, \mathrm{thr^{- }}$, and $l e \mathrm{u}^{-}$denote that strains require respectively thiamine, methionine, proline, threonine, and leucine for growth; $\operatorname{lac}^{-}\left(z_{1}^{-}\right), \operatorname{lac}^{-}\left(y^{-}\right)$, unable to utilize lactose as carbon source (respectively, unable to produce the lactose-splitting enzyme $\beta$-galactosidase, and unable to concentrate lactose within the cell); $l a c^{+}$, able to utilize lactose as carbon source; $\boldsymbol{S}^{s}, \boldsymbol{S}^{r}$, respectively sensitive and resistant to streptomycin.

\section{Conditions of growth.}

Inocula from minimal media were taken from $15 \mathrm{hr}$ limiting-glucose cultures, previous experience in this laboratory having indicated that these give the most reproducible patterns of growth. Cultures to be tested for ability to grow in the absence of a particular growth factor were centrifuged for $10 \mathrm{~min}$. at $3000 \mathrm{rev} . / \mathrm{min}$. and washed once with phosphate buffer $\left(\mathrm{Na}_{2} \mathrm{HPO}_{4}\right.$. $12 \mathrm{H}_{2} \mathrm{O}, 16 \mathrm{~g}$.; $\mathrm{KH}_{2} \mathrm{PO}_{4}$, 2.95 g.;

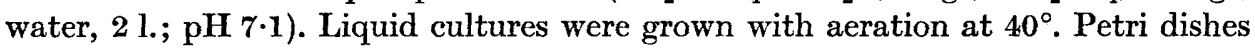
containing the solid media were incubated at $37^{\circ}$.

\section{Estimation of cultures.}

Liquid cultures were estimated turbidimetrically by use of a Hilger 'Spekker' light absorptiometer. The mass/unit volume of a sample was expressed in arbitrary units, actually the number of bacteria $/ \mathrm{ml}$. in that dilution giving the same reading as the sample, of a known culture used to calibrate the instrument. Lag and mean generation time (MGT) were calculated from the growth curve as described by Hinshelwood (1946, p. 37). Colony diameters on solid media were measured under a Watson $\times 25$ stereoscopic microscope equipped with a scale eye-piece. 


\section{Technique of crossing.}

The parent cultures (from stock cultures in nutrient media unless otherwise specified) were inoculated into broth and incubated overnight (about $15 \mathrm{hr}$ ) at $37^{\circ}$. They were then diluted 10 times into fresh broth and aerated at $40^{\circ}$ for $1 \frac{1}{2} \mathrm{hr}$, at which point all strains could be shown to be growing exponentially and had reached counts of the order of $10^{8}$ bacteria $/ \mathrm{ml}$. Equal volumes of the broth cultures were then mixed and incubated without aeration for $2 \mathrm{hr}$ at $40^{\circ}$. The mixture and samples of the parent cultures were centrifuged for $10 \mathrm{~min}$. at $3000 \mathrm{rev} . / \mathrm{min}$., washed once with phosphate buffer, and diluted in $0.85 \%$ saline for plating. The resuspended parent organisms were plated on the selecting medium, to investigate possible reversion, and at high dilution on FSM agar, to obtain single colonies.

Single recombinant colonies were picked after $48 \mathrm{hr}$ and were grown up twice on medium corresponding to the selecting plate, to free them from parental contamination, before testing for unselected markers. Single colonies of the parent strains were grown up for testing on FSM media.

Characterization of lac and pro markers in parents and recombinants.

The growth in lactose of the $\mathrm{lac}^{-}$strains studied here takes place, if at all, after a long lag, and is then frequently non-logarithmic and erratic. Such growth has therefore been characterized by the time taken to grow from a standard initial mass to an arbitrary standard mass; the time required to grow from a mass $10^{x} / \mathrm{ml}$. to a mass $10^{y} / \mathrm{ml}$. will be denoted by $T_{x}^{y}$. Histograms showing the distribution of $T$ for single-colony isolates of a strain are, under controlled conditions, characteristic of the lesion affecting lactose utilization that it bears. This is true whether growth is enabled to occur by physiological adaptation (for evidence that this may be involved in growth on lactose media of the naturally occurring $y^{-}$' mutabile' strain of Escherichia coli, see Dean \& Hinshelwood, 1954, 1956, 1959), or by the growth of $\mathrm{lac}^{+}$mutants; in the latter case the histogram will be affected, for instance, by the rate of occurrence of different mutant types and their growth rates under the conditions involved. Such histograms obtained for recombinant isolates can then be compared with those for the parent strains.

Strains 75 and 751 grew in proline-less media without a lag, but with an MGT of about $3 \mathrm{hr}$, as compared to 45-60 min. for wild-type Escherichia coli k-12. These strains, and recombinants showing the same growth pattern, were characterized by MGT. Recombinants showing a different pattern of growth in prolineless media will be discussed below.

\section{RESULTS}

\section{Reversion of selected markers}

No growth was ever observed on inoculation from a culture of strain 75 or 751 in medium containing methionine into liquid or solid medium lacking this amino acid. Liquid cultures lacking threonine or leucine, inoculated with strain $80 \mathrm{R}$ grown in FSM medium, showed slow and erratic (non-logarithmic) growth in 1-3 or 3-6 days respectively. Growth of such inocula on similarly constituted solid media differed considerably according as the absent amino acid was threonine or leucine. On 
threonine-less medium, colonies were observed to develop continuously from the time (dependent on inoculum size) at which the first ones appeared, until the cessation of growth through plate exhaustion, at which point a continuous gradation of colony sizes was found. On leucine-less medium, on the other hand, a constant small fraction $\left(c .1\right.$ in $\left.5 \times 10^{7}\right)$ of bacteria plated gave rise to 'primary' colonies, which grew at the normal rate for K-12 strains on FSM media, i.e. reached a diameter of $1 \mathrm{~mm}$. in 1-2 days. After 4-5 days, 'secondary' colonies, showing the continuous development noted for growth on threonine-less medium, began to appear. The gap between the appearances of 'primary' and 'secondary' colonies led to a characteristic bimodality in histograms of colony size distribution till at least the sixth day from plating. It might be suggested that 'primary' colonies develop from leu ${ }^{+}$organisms pre-existing in the inoculum, whereas 'secondary' colonies develop as a result of events (mutation during early 'background' growth or physiological adaptation) taking place only after plating; however, it is not necessary to postulate such an inhomogeneity in the inoculum population (see Dean \& Hinshelwood, 1956).

On medium lacking both threonine and leucine, 'primary' colonies developed as on medium lacking leucine alone, but the formation of 'secondary' colonies was drastically decreased.

These growth factor requirements were found to be stable on continued subculture in broth or in FSM media so constituted that exhaustion of a required compound was never the cause of cessation of growth (media 'complete' for required growth factors). On subculture of strain $80 \mathrm{R}$ in media 'incomplete' for one or both of these amino acids, the requirement for the compound(s) present in limiting amount was gradually lost.

\section{Mutation to streptomycin resistance}

Cultures of strains Hfr $\mathrm{H} 59$ and Hfr R1, when plated on broth or FSM media containing streptomycin, gave rise to colonies corresponding to one organism in about $10^{8}$ inoculated, which appeared within 2 days. No later-developing colonies were ever observed.

\section{Growth of recombinants on selecting media}

Recombinant colonies developed as rapidly on the selecting media as did parental colonies on their FSM media. Later colony development on the selecting plates was not observed, suggesting that transfer of selected markers to recombinants was an all-or-nothing matter.

The minimum yield of recombinants obtained in an $\mathrm{F}^{+} \times \mathrm{F}^{-}$cross was of the order of 100 colonies on plating $\mathbf{0 . 3} \mathrm{ml}$. of the undiluted washed mating mixture. It therefore seems probable, from the above, that the chance that a revertant or streptomycin-resistant mutant was included among the recombinant isolates in the experiments to be described was never more than 1 in 100 (and, of course, was much lower in the high-frequency crosses).

Crossing experiments, and expression of unselected markers in parents and recombinants

Cross (1): $H$ fr $R 1 \times 75$. In this cross, strain 75 acted as acceptor and Hfr $\mathbf{R}$ as donor. The latter strain transfers $\mathrm{met}^{+}$with high frequency, and $\mathrm{lac}^{+}$and $\mathrm{pro}^{+}$with considerably lower frequency (de Haan \& Gross, 1962), so that, with proline- 
thiamine-streptomycin agar as selecting medium, a high proportion of the $\mathrm{met}^{+} \boldsymbol{S}^{r}$ recombinants should be lac $^{-}$or pro-.

The patterns of growth of parent and recombinant isolates are illustrated in Fig. 1. Of the 90 recombinants tested, 88 showed the same characteristics of growth in lactose medium as the $l_{a c^{-}}$parent. Of the other two, one (no. 77) grew similarly to the $\mathrm{lac}^{+}$parent; the other (no. 48) grew only after a lag of about $12 \mathrm{hr}$, but thereafter exponentially, with an MGT of about $2 \mathrm{hr}$.

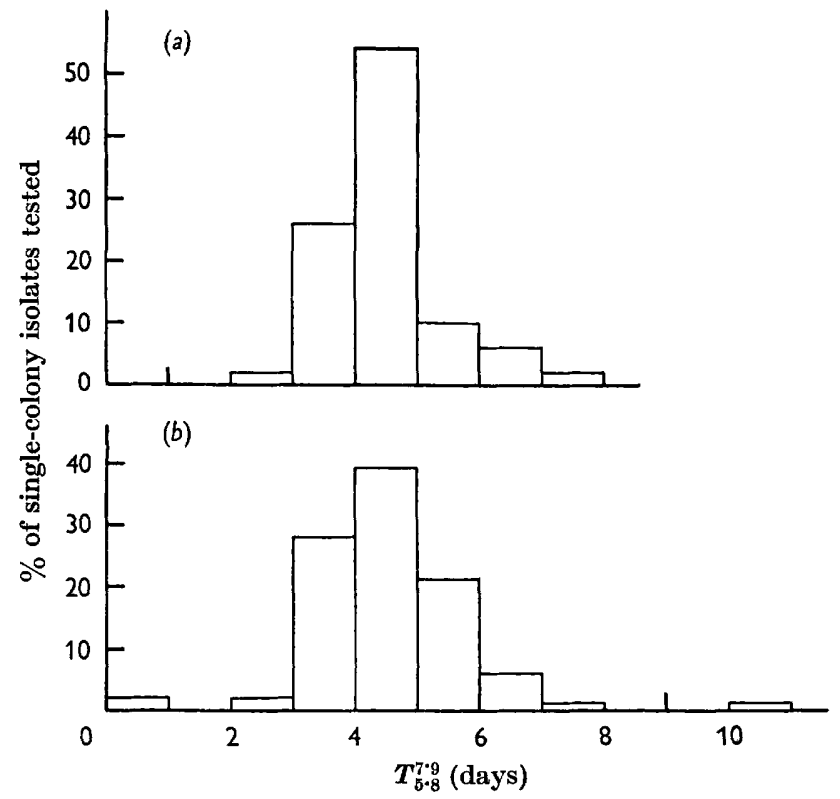

Fig. 1. Histograms of $T_{5: 8}^{7 \cdot 9}$ (the time required for bacterial mass to increase from $10^{5.8}$ to $10^{7 \cdot 9}$ units $/ \mathrm{ml}$.) for first growth in FSM lactose medium of parent and recombinant colony isolates from cross (1). (a) Isolates of parent strain 75:50 colonies tested. (b) Recombinant isolates: 90 colonies tested.

All the recombinants tested grew in proline-less medium similarly to the proparent, with the exception of no. 77, which behaved as the wild type. In this experiment, therefore, no signs could be detected of a continuous variation among recombinants between the lac and pro characters of the parent strains. However, one intermediate lac type, no. 48, was found. Since this recombinant differed from the $\mathrm{lac}^{+}$pro $^{+}$no. $\mathbf{7 7}$ in being pro $^{-}$, it was thought possible that this might correlate with the anomalous lac character; however, a number of pro $^{+}$revertants tested showed no improved growth in lactose medium.

Cross (2): $751 \times 80_{R}$. In this cross, strain 751 was used as donor and $80 \mathrm{R}$ as acceptor, proline+thiamine agar being the selecting medium. The former strain, being $\mathbf{F}^{+}$, is capable of transferring any selected marker at low frequency (Jacob \& Wollman, 1961, p. 156); when the selected markers are $t h r^{+} l e u^{+}$, some $50 \%$ of recombinants may be expected to inherit the pro- character of the donor, and a slightly lower percentage its $\mathrm{lac}^{-}$character.

The results of the cross were as follows. Of the 54 recombinants tested, 24 were 
found to be $\mathrm{lac}^{+} \mathrm{pro}^{+}, 8 \mathrm{lac}^{+} \mathrm{pro}^{-}$, and $22 \mathrm{lac}^{-} \mathrm{pro}^{-}$. The '+' characters in all cases resembled those of the parent; but the '-' characters differed considerably. None of the $l a c^{-}$recombinants grew, from the standard inoculum, in lactose medium (15 days being allowed before non-growing cultures were rejected). In proline-less medium, the pro $^{-}$recombinants showed a slight initial mass increase, followed by a lag which gave way to further growth only after at least $12 \mathrm{hr}$. The lac $^{+}$pro $^{-}$

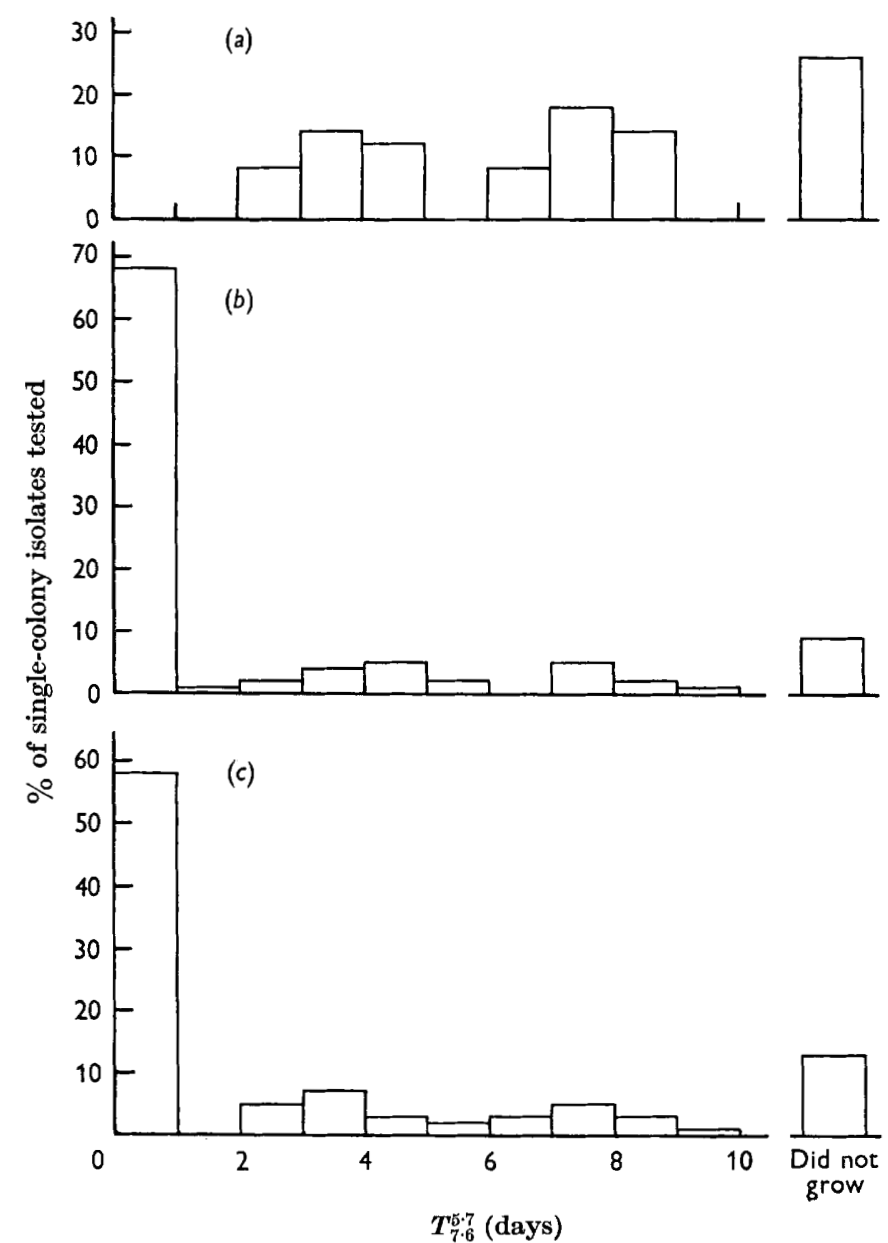

Fig. 2. Histograms of $T_{5 \cdot 7}^{7 \cdot 6}$ (the time required for bacterial mass to increase from $10^{5 \cdot 7}$ to $10^{7.6}$ units $/ \mathrm{ml}$.) for first growth in FSM lactose medium of parent and recombinant colony isolates from cross (3). (a) Isolates of parent strain Hfr H 59: 50 colonies tested. The corresponding histogram for the $\mathrm{F}^{+} 59$ parent is similar. (b) Recombinant isolates from cross with $\mathrm{Hfr} \mathbf{H 9}$ as donor: 70 colonies tested. (c) Recombinant isolates from cross with $\mathbf{F}+\mathbf{5 9}$ as donor: 70 colonies tested.

recombinants showed the $\mathrm{lac}^{+}$characteristies of the $80 \mathrm{R}$ parent, and the pro $^{-}$characteristics of the $\mathrm{lac}^{-}$pro- $^{-}$recombinants, indicating that the change in pro $^{-}$character does not depend on the presence of the $l a c^{-}$character.

Cross (3): $59 \times 80 \mathrm{R}$. In order to ascertain that the above results are not due to 
some general effect whereby the reversion of a '-' mutation to wild type is in some way hindered after passing from a donor strain into a recombinant, a cross was performed with a $\mathrm{lac}^{+}$recipient and the $z_{1}{ }^{-}$donor 59. The cross was performed in duplicate, the donor strain in one case being the normal Hfr from stock culture, and in the other a substrain $\left(59 \mathrm{~F}^{+}\right)$which had lost the Hfr characteristic during 29 subcultures in FSM glucose medium. The selecting medium was thiamine-streptomycin agar.

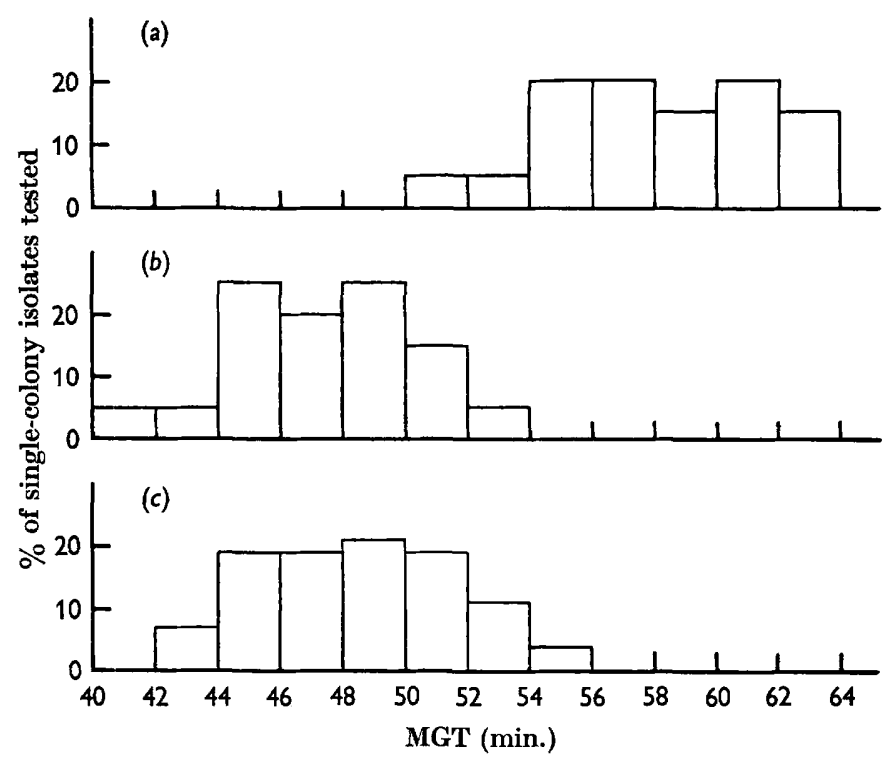

Fig. 3. Histograms of mean generation times in lactose medium containing threonine, leucine, proline and thiamine, of parent and recombinant isolates from cross (4). (a) $\mathrm{Lac}^{+}$recombinants from cross (4) $b$ ( $\mathrm{lac}^{+}$recipient parent not trained to lactose): 19 isolates tested. (b) $\mathrm{Lac}^{+}$parent strain $80 \mathrm{~m}$ from cross (4) $a$ (trained to lactose): 20 isolates tested. (c) $\mathrm{Lac}^{+}$recombinants from cross (4) a ( $\mathrm{lac}^{+}$recipient parent trained to lactose): 24 isolates tested.

The results of the cross are illustrated in Fig. 2. It is seen that the $T$ distribution for growth in lactose medium, of the lac $^{-}$recombinants resembles in both cases that of the $l a c^{-}$parent. The results of cross (2) are therefore probably specific to the characters concerned.

Cross (4): $751 \times 80_{R}$ trained to maximum growth rate in lactose medium. Strain $80 \mathrm{R}$, subcultured many times in FSM glucose medium, grows on first subculture in FSM lactose medium with an MGT of 55-72 min. On continued subculture in this medium, the MGT gradually decreases, with erratic fluctuations, finally becoming stabilized in the region $45-50 \mathrm{~min}$.

It was decided to investigate how the capacity for growth in lactose medium at the maximum rate is transmitted to recombinants; whether $\mathrm{lac}^{+}$recombinants from a cross with a highly trained $80 \mathrm{R}$ parent grow at the maximum rate, at the untrained rate, in some intermediate range, or whether a complete spectrum of MGT's is found.

Cross (4) was performed similarly to cross (2), but in duplicate. With the 751 parent as before, two $80 \mathrm{R}$ parent strains were used; one subcultured 35 times in 
FSM lactose medium (cross $a$ ), the other, providing a control, subcultured the same number of times in FSM glucose medium (cross $b$ ).

28 recombinants from cross $a$ and 25 from cross $b$ were examined, together with $20 \mathrm{lac}^{+}$parent colonies from cross $a$. Of the recombinants from cross $a, 15$ were found to be $\mathrm{lac}^{+} \mathrm{pro}^{+}, 9 \mathrm{lac}^{+} \mathrm{pro}^{-}$, and $4 \mathrm{lac}^{-} \mathrm{pro}^{-}$; of those from cross $b, 16$ were $\mathrm{lac}^{+} \mathrm{pro}^{+}, 3 \mathrm{lac}^{+} \mathrm{pro}^{-}$, and $6 \mathrm{lac}^{-}$pro $^{-}$. Histograms showing the MGT distributions of the $\mathrm{lac}^{+}$recombinants and of the $20 \mathrm{lac}^{+}$parent isolates from cross $a$ are shown in Fig. 3; MGT's were measured in threonine+leucine+proline+thiamine lactose medium, to eliminate variations due to differences in the degree of supplementation of the test medium. It is seen that the distribution of MGT's shown by the recombinants from cross $a$ resembles strongly that shown by the trained $l a c^{+}$parent.

It was also found that the $l a c^{-}$recombinants from cross (4) $a$ were able to grow, from the standard inoculum, in proline + thiamine lactose medium, although the $T$ distribution extended to longer times (10 days in some cases) than in the case of the $l a c^{-}$parent. Those from cross $b$ behaved in all respects like those from cross (2).

The pro characteristics of the recombinants from crosses $a$ and $b$ resembled those from cross (2), except in that one $\mathrm{lac}^{+} \mathrm{pro}^{-}$recombinant from cross $b$ showed the form of growth, in proline-less medium, of the pro- $^{-}$parent, with no lag and an MGT of about $3 \mathrm{hr}$.

\section{DISCUSSION}

The results given above, in their broad outline, are consistent with the theory that the lac and pro characters are controlled in Escherichia coli $\mathrm{K}-12$ by particulate determinants between which no merging occurs, rather than by the continuously varying levels in cellular reaction networks. In only one case (recombinant no. 48 from cross (1)) can it be said that an intermediate between the parental characteristics was observed. However, if the fine details of recombinant behaviour are considered, there remain certain observations for which to account.

The sharply reduced ability to revert to wild-type behaviour shown by the lac and pro- recombinants from crosses (2) and (4) $b$ can be explained by postulating the existence of a gene, powerfully affecting the frequencies of certain mutations, present in different allelic forms in strains 751 and $80 \mathrm{R}$; the allele in strain $80 \mathrm{R}$ being presumed to reduce the rates of these particular $\mathrm{lac}^{-} \rightarrow \mathrm{lac}^{+}$and $\mathrm{pro}^{-} \rightarrow$ pro $^{+}$ mutations below those allowed by the allele present in strain 751. To explain why the allele present in $\mathbf{7 5 1}$ is found so rarely among the recombinants studied (once only-in the $\mathrm{lac}^{+} \mathrm{pro}^{-}$recombinant from cross (4) $b$ showing the pro $^{-}$characteristics of strain 751), it must be supposed that it is transmitted only at low frequency under the conditions of crosses (2) and (4). This could occur if the gene were located, for instance, between the his and thi loci (according to the linkage maps of Jacob \& Wollman, 1961, p. 165, and Hayes, 1964, p. 567). The function of such a gene would be of considerable interest.

However, according to the theory of Dean \& Hinshelwood, to which reference has already been made, it is possible that the disorder caused in the recipient cell by the introduction of fragments from the donor cell might well have some heritable effect. These authors picture the steady-state values of the various cellular metabolic pathways during exponential growth as necessarily being delicately balanced with respect to one another, to permit the optimal growth rate. The incorporation of 
extraneous material following conjugation may affect the quantitative interrelationships of some of these pathways, yielding a transient unbalanced state which might, however, persist for many generations. The cell, while in this disorganized state, could show an impaired capacity for the automatic adjustments needed in adapting to changes in the environment. From this standpoint, therefore, the reduced reversion rates of $\mathrm{lac}^{-}$and $\mathrm{pro}^{-}$recombinants (if such reversion is assumed to take place by a process of physiological adaptation rather than by mutation) would be expected. A similar observation with another method of introducing disorder-ultra violet irradiation-has been reported by E. M. Lederberg (1952).

The improvement in growth rate of $\mathrm{lac}^{+}$strains on continued subculture in lactose media may be explained either in terms of overgrowth of the typical organisms of the initial culture by mutants, originally present in low proportion but capable of the faster growth rate, or of a process of adaptation on the part of the majority of bacteria in the population. The results of cross (4) $a$ suggest that, if the determinants of the faster growth rate are chromosomal, either their alleles in strain $\mathbf{7 5 1}$ are transferred with very low frequency, e.g. because the genes lie in the his-thi region, or the genes are closely linked to or lie within the lac region. The possibility that mutations inside the lac region are involved clearly merits further biochemical and genetic investigation. If this is the case, then the system clearly differs from that involved in the training to increasingly high degrees of drug resistance cited above, where the only hypothesis consistent with the genetic data is that of a number of mutations at loci widely distributed over the bacterial chromosome. On the physiological adaptation hypothesis, it would be quite expected that adaptation to improved growth in lactose medium involves modification of the lac determinant, so that the two appear to be transferred together.

The increased rate of the $\mathrm{lac}^{-} \rightarrow \mathrm{lac}^{+}$change in $l a c^{-}$recombinants from cross (4)a can be explained only by the hypotheses (i) that the loci concerning training to lactose are situated considerably beyond the lac region, as suggested above, so that the alleles present in the lactose-trained recipient are present in most $l_{a c^{-}}$as well as $\mathrm{lac}^{+}$recombinants, and (ii) that these loci also affect this particular mutation rate. It is not easy to see why (ii) should be the case. On the other hand, from the physiological adaptation viewpoint, it is reasonable that a cytoplasmically inherited lactose 'training' may improve the capacity of the cells to adapt from lacto $\mathrm{lac}^{+}$.

The author wishes to thank Sir Cyril Hinshelwood, O.M., F.R.S., for much helpful discussion and advice during the course of this work, and Dr A. C. R. Dean for many practical suggestions. $\mathrm{He}$ is also indebted to the Department of Scientific and Industrial Research for the award of a Research Studentship.

\section{REFERENCES}

Bartlett, G. W. \& Hinshelwood, C. N. (1959). Observations on the drug resistance of bacterial recombinants. Proc. R. Soc. B, 150, 318.

Cavalli, L. L. \& Maccacaro, G. A. (1950). Chloromycetin resistance in Escherichia coli, a case of quantitative inheritance in bacteria. Nature, Lond. 166, 991.

Cavalui, L. L. \& Maccacaro, G. A. (1952). Polygenic inheritance of drug-resistance in the bacterium Escherichia coli. Heredity, 6, 311. 
Dean, A. C. R. \& Hinshelwood, C. N. (1954). The adaptation of Bacterium coli mutabile to lactose. Proc. $R$. Soc. B, 142, 225.

Dean, A. C. R. \& Hinshelwoon, C. N. (1956). The statistics of colony development by Bacterium coli mutabile on lactose agar. Proc. R. Soc. B, 146, 109.

Dean, A. C. R. \& Hinshelwood, C. N. (1959). Further observations on the adaptation of coliform bacteria to sugars. Proc. R. Soc. B, 151, 435.

Dean, A. C. R. \& Hinshelwood, C. N. (1964a). Some basic aspects of cell regulation. Nature, Lond. 201, 232.

Dean, A. C. R. \& Hinshelwood, C. N. (1964b). What is heredity? Nature, Lond. 202, 1046.

Demerec, M. (1948). Origin of bacterial resistance to antibiotics. J. Bact. 56, 63.

DE HAan, P. G. \& Gross, J. D. (1962). Transfer delay and chromosome withdrawal during conjugation in Escherichia coli. Genet. Res. Camb. 3, 188.

Hayes, W. (1964). The Genetics of Bacteria and their Viruses. Oxford: Basil Blackwell.

Hinshelwood, C. N. (1946). The Chemical Kinetics of the Bacterial Cell. Oxford: Clarendon Press.

Jacob, F. \& Wollman, E. L. (1961). Sexuality and the Genetics of Bacteria. New York: Academic Press.

Lederberg, E. M. (1952). Allelic relationships and reverse mutation in Escherichia coli. Genetics, 37, 469.

LEDERBERG, J. (1947). Gene recombination and linked segregations in Escherichia coli. Genetics, 32, 505. 